\title{
Disorder and dynamics of free and caged molecules in crystals
}

\author{
Guanqun Cai ${ }^{1}$, Franz Demmel ${ }^{2}$, Richard Dixey ${ }^{1}$, Bernet E. Meijer ${ }^{1}$, Shurong Yuan ${ }^{1}$, \\ Helen C. Walker ${ }^{2}$ and Anthony E. Phillips ${ }^{1}$
}

\author{
${ }^{1}$ School of Physics and Astronomy, Queen Mary University of London, London E1 4NS, United Kingdom., \\ ${ }^{2}$ ISIS Neutron and Muon Source, Rutherford Appleton Laboratory, Harwell Oxford OX11 0QX, United Kingdom \\ a.e.phillips@qmul.ac.uk
}

\begin{abstract}
Molecules and molecular ions, unlike individual atoms, have rotational degrees of freedom. This simple observation means that they can be particularly good building blocks for disordered crystal structures. Such behaviour is not merely a crystallographic curiosity: it is responsible for many important materials properties. For instance, if, in some material, a molecule with a permanent dipole moment is free to rotate at high temperatures but freezes into place at low temperatures, the two phases will have different entropies and dielectric constants. The result will be a dielectric switching material; it is likely to be an electro- and/or barocaloric, where the phase transition responds to an external electric field or pressure; and it may also be pyro- and even ferroelectric if the low-symmetry phase is polar.
\end{abstract}

Studying such materials requires a combination of experimental and computational techniques. Traditional crystallographic methods remain vital, but entail a time and space average that can obscure the behaviour of the disordered phase. Thus it is also important to use methods such as total scattering, which are sensitive to local deviations from that average; and to study also the dynamics, or how structures change over time. Neutron scattering methods are especially appropriate because they reveal the behaviour of hydrogen atoms, which is essential to understanding these rotating molecules, and provide both structural and dynamic information.

A particular focus of recent interest has been the family of molecular perovskites, in which molecular ions on the "A" site almost always display this sort of order-disorder transition [1]. This site is a cubic interstice of the perovskite framework, which provides both structural stability and the freedom for the ions to rotate. But of course order-disorder behaviour does not require this specific coordination framework, or indeed any framework at all: similar molecular "scaffolding" can be provided by other weak interactions, including van der Waals and hydrogen bonding.

Here we compare "free" to "caged" molecules and molecular ions that undergo entropic transitions. We consider the cyanide-bridged elpasolite (double perovskite) analogues $\left(\mathrm{C}_{3} \mathrm{H}_{5} \mathrm{~N}_{2}\right)_{2} \mathrm{~K}\left[\mathbf{M}(\mathrm{CN})_{6}\right], \mathrm{C}_{3} \mathrm{H}_{5} \mathrm{~N}_{2}=$ imidazolium, $\mathbf{M}=\mathrm{Fe}$, Co [2]; the molecular material adamantane, $\mathrm{C}_{10} \mathrm{H}_{16}$ [3], and the molecular-ionic compound ammonium sulfate, $\left(\mathrm{NH}_{4}\right)_{2} \mathrm{SO}_{4}$ [4]. We have studied these materials' structure by Bragg and total neutron scattering, and their dynamics by inelastic and quasielastic neutron scattering, complemented by density-functional theory simulations. Combining these methods provides a detailed picture of the actual rotational and vibrational freedom that molecules have in these materials, and hence of the structural origins of their useful properties. In particular, we show that the limiting cases of free rotation and harmonic oscillation can both be inaccurate and even seriously misleading, with the true situation lying somewhere between these extremes. Our results will direct future attempts at "entropic engineering": designing molecular materials to have specific order-disorder behaviour.

[1] Kieslich, G. \& Goodwin, A. L. (2017). Mater. Horiz. 4, 362-366.

[2] Duncan, H. D., Beake, E. O. R., Playford, H. Y., Dove, M. T. \& Phillips, A. E. (2017). CrystEngComm 19, 7316-7321; Phillips, A. E. \& Fortes, A. D. (2017). Angew. Chem. Int. Ed. 56, 15950-15953; Phillips, A. E., Cai, G. \& Demmel, F. (2020). Chem. Commun. 56, $11791-11794$.

[3] Beake, E. O. R., Tucker, M. G., Dove, M. T. \& Phillips, A. E. (2017). ChemPhysChem 18, 459-464.

[4] Cai, G. (2020). Studying orientational disorder with neutron total scattering, Ph.D. thesis, Queen Mary University of London, U.K. Keywords: disorder; total neutron scattering; quasielastic neutron scattering; inelastic neutron scattering; framework
materials 